PROCEEDINGS OF THE

AMERICAN MATHEMATICAL SOCIETY

Volume 135, Number 10, October 2007, Pages 3233-3240

S 0002-9939(07)09030-2

Article electronically published on June 22, 2007

\title{
ON THE MODULI OF CONVEXITY
}

\author{
A. J. GUIRAO AND P. HÁJEK
}

(Communicated by Jonathan M. Borwein)

\begin{abstract}
It is known that, given a Banach space $(X,\|\cdot\|)$, the modulus of convexity associated to this space $\delta_{X}$ is a non-negative function, nondecreasing, bounded above by the modulus of convexity of any Hilbert space and satisfies the equation $\frac{\delta_{X}(\varepsilon)}{\varepsilon^{2}} \leq 4 L \frac{\delta_{X}(\mu)}{\mu^{2}}$ for every $0<\varepsilon \leq \mu \leq 2$, where $L>0$ is a constant. We show that, given a function $f$ satisfying these properties then, there exists a Banach space in such a way its modulus of convexity is equivalent to $f$, in Figiel's sense. Moreover this Banach space can be taken to be two-dimensional.
\end{abstract}

\section{INTRODUCTION}

In this paper we are concerned with the basic properties of the modulus of convexity of a Banach space which is defined as follows.

Definition 1.1 (Modulus of convexity). The modulus of convexity of $(X,\|\cdot\|)$ is the function that for each $\varepsilon \in[0,2]$ takes the value

$$
\delta_{X}(\varepsilon)=\inf \{1-\|x+y\| / 2:\|x\|=\|y\|=1, \quad\|x-y\|=\varepsilon\} .
$$

The following result concerning moduli of convexity was established by Figiel $[3]$.

Proposition 1.2. The modulus $\delta_{X}$ satisfies the following properties:

- $\delta_{X}(\cdot)$ is non-decreasing.

- If $0<\varepsilon \leq \mu$, then for a universal constant $0<L<3.18$,

$$
\frac{\delta_{X}(\varepsilon)}{\varepsilon^{2}} \leq 4 L \frac{\delta_{X}(\mu)}{\mu^{2}} .
$$

Additionally, due to some results by G. Nordlander [4, we can add another property to the previous proposition, which is the following result:

Proposition 1.3. For every $\varepsilon \in[0,2]$ we have $\delta_{X}(\varepsilon) \leq \delta_{E}(\varepsilon)$.

Received by the editors June 26, 2006.

2000 Mathematics Subject Classification. Primary 46B03.

Key words and phrases. Banach spaces, modulus of convexity, uniformly rotund norms.

The first author was supported by grants MTM2005-08379 of MECD (Spain), 00690/PI/04 of Fundación Séneca (CARM, Spain), and AP2003-4453 of MECD (Spain).

The second author was supported by the Institutional Research Plan, AV0Z10190503 and A100190502.

(C)2007 American Mathematical Society Reverts to public domain 28 years from publication 
Here, $\delta_{E}$ is the modulus of convexity of any Hilbert space. Obviously $\delta_{E}=\delta_{l_{2}}$, and from the parallelogram identity, it can be easily determined that

$$
\delta_{E}(\varepsilon)=1-\sqrt{1-\frac{\varepsilon^{2}}{4}} .
$$

In the following section we will show that these conditions are enough for a function to be a modulus of convexity.

\section{Characterization of moduli of CONVEXity}

Following the article [3], we will consider the following concept of equivalence between functions.

Definition 2.1. Given two non-decreasing functions $f$ and $g$, each one defined on a segment $[0, a]$, let us write $f \prec g$ if there exist positive constants $A, B, C$ such that $A f(B t) \leq g(t)$ for $t \in[0, C]$; we shall consider $f$ and $g$ as equivalent, denoted by $f \sim g$, iff $f \prec g \prec f$.

Clearly, $\prec$ defines a partial ordering in the space of non-decreasing functions on $[0, a]$, and it is easy to verify that $\sim$ is indeed an equivalence relation. In particular, it is consistent to use $\sim$ also for the whole equivalence classes of functions. It is rather standard to construct two non-comparable functions (classes) so $\prec$ is not a linear ordering. Now we can consider all those functions that satisfy the properties described in the Introduction. Indeed we state the following definition:

Definition 2.2. A "modulus function" is a non-negative real function $f$ defined on a segment $[0, a]$ and satisfying the following conditions:

(1) $f(\varepsilon) \leq \delta_{E}(\varepsilon)$.

(2) $f(\cdot)$ is non-decreasing.

(3) If $0<\varepsilon \leq \mu$, then for a positive constant $L$,

$$
\frac{f(\varepsilon)}{\varepsilon^{2}} \leq L \frac{f(\mu)}{\mu^{2}} .
$$

Our main result states that a function is a modulus function if and only if there exists a Banach space $X$. In fact, we may choose $X=l_{2}^{2}$ and a new norm in such a way that the modulus of convexity of the new norm is equivalent to the function. In order to show that result, we need to do some preliminary work in relation to the general construction of the norm.

2.1. Construction. Let $\left(\mathbb{R}^{2},\|\cdot\|\right)$ be the canonical two-dimensional real Banach space where $\|\cdot\|$ is the Euclidean norm, and let $S=\left\{x \in \mathbb{R}^{2}:\|x\|=1\right\}$ be the unit sphere, that is, the circle of radius one and whose center is the origin.

Let us take any point in $S$, say $a_{1}$, its symmetric $-a_{1}$, and a positive value $\varepsilon$. Starting in $a_{1}$ and $-a_{1}$, we will take in the clockwise direction on $S$ points $b_{1}, a_{2}$, $-b_{1},-a_{2}$ such that the segments $\left[a_{1}, b_{1}\right]$ and $\left[b_{1}, a_{2}\right]$ have length $\varepsilon$. Now, we repeat the same process starting at $a_{2}$ but in such a way that the length of the segments are $\varepsilon^{2}$. Let us suppose that we have already chosen $\left\{\left(a_{i}, b_{i}\right)\right\}_{i=1}^{n}$ and $a_{n+1}$; then we take points $b_{n+1}$ and $a_{n+2}$ such that the segments $\left[a_{n+1}, b_{n+1}\right]$ and $\left[b_{n+1}, a_{n+2}\right]$ have length $\varepsilon^{n+1}$.

It is a crucial fact that the family $\left\{\left(a_{i}, b_{i}\right)\right\}_{i \in \mathbb{N}}$ lies just in one hemisphere of the sphere $S$. Thus in order to clarify the size that $\varepsilon$ can take, we have the following result: 
Lemma 2.3. Given two points $x$ and $y$ of the unit sphere $S$, the angle $\alpha$ opposite to the segment $[x, y]$ of the triangle with extremes $x, y$, and the origin, takes the value

$$
\alpha=2 \arcsin \left(\frac{\|x-y\|}{2}\right) .
$$

Then, in the previous construction, we have that the segments we have chosen cover an angle with arc length

$$
4\left(\sum_{n \in \mathbb{N}} \arcsin \left(\frac{\varepsilon^{n}}{2}\right)\right),
$$

and if $\varepsilon \leq 1 / 2$, it is less than $\pi$.

Proof. All the results but the last are obvious. In order to show that for $\varepsilon \leq 1 / 2$, we have

$$
4\left(\sum_{n \in \mathbb{N}} \arcsin \left(\frac{\varepsilon^{n}}{2}\right)\right) \leq \pi
$$

Let us note that the function $x / \sin (x)$ when $x \leq 0.34$ is bounded above by 1.02 , and then when $\frac{\varepsilon^{n}}{2} 1.02 \leq \frac{1}{3}$,

$$
\arcsin \left(\frac{\varepsilon^{n}}{2}\right) \leq \arcsin \left(\sin \left(\frac{\varepsilon^{n}}{2} 1.02\right)\right)=\frac{\varepsilon^{n}}{2} 1.02,
$$

since arcsin is an increasing function. Then for $\varepsilon \leq 1 / 2$ we have the above estimate and

$$
4\left(\sum_{n \in \mathbb{N}} \arcsin \left(\frac{\varepsilon^{n}}{2}\right)\right) \leq 4\left(\sum_{n \in \mathbb{N}} \frac{\varepsilon^{n}}{2} 1.02\right) \leq 2.04 \sum_{n \in \mathbb{N}} \varepsilon^{n}=2.04 \frac{\varepsilon}{1-\varepsilon} \leq 2.04 .
$$

But obviously this value is less than $\pi$.

Now we can be assured that our selection of points is good in the sense that they do not overlap each other with their symmetric points.

We are going to define a new unit sphere for $\mathbb{R}^{2}$. In order to do this, we consider a fixed $i \in \mathbb{N}$, take the segment $\left[a_{i}, b_{i}\right]$, delete from $S$ the points of the arc lying between $a_{i}$ and $b_{i}$, and replace them with a parabolic arc. In order to specify these parabolic arcs, we will use a new affine coordinate system.

Indeed, given a non-negative value $c_{i}$, let us consider the system of coordinates defined by the following equation:

$$
\left(\begin{array}{l}
x^{i} \\
y^{i}
\end{array}\right)=\frac{-1}{\sqrt{4-\varepsilon^{2 i}}}\left(\begin{array}{cc}
a_{i}^{\prime \prime}+b_{i}^{\prime \prime} & a_{i}^{\prime}+b_{i}^{\prime} \\
-a_{i}^{\prime}-b_{i}^{\prime} & a_{i}^{\prime \prime}+b_{i}^{\prime \prime}
\end{array}\right)\left(\begin{array}{l}
x \\
y
\end{array}\right)+\left(\frac{1}{2}+\frac{c_{i}}{\sqrt{4-\varepsilon^{2 i}}}\right)\left(\begin{array}{c}
a_{i}^{\prime}+b_{i}^{\prime} \\
a_{i}^{\prime \prime}+b_{i}^{\prime \prime}
\end{array}\right),
$$

where $a_{i}=\left(a_{i}^{\prime}, a_{i}^{\prime \prime}\right)$ and $b_{i}=\left(b_{i}^{\prime}, b_{i}^{\prime \prime}\right)$ are expressed in the canonical system of coordinates. It happens that the $y^{i}$-axis contains both the initial origin of coordinates and the midpoint of the segment $\left[a_{i}, b_{i}\right]$. Therefore, the origin of the new system will lie on the $y^{i}$-axis, at distance $c_{i}$ from the segment $\left[a_{i}, b_{i}\right]$ (see Figure 1).

Thus the old arc is replaced by the set $\left\{\left(t, y_{i}(t)\right):|2 t| \leq \varepsilon^{i}\right\}$ (expressed in terms of the system $\left.\left(x^{i}, y^{i}\right)\right)$, where $y_{i}$ is the function defined by $y_{i}(t)=\left(4 c_{i} / \varepsilon^{i}\right) t^{2}$.

For the symmetric segment $\left[-a_{i},-b_{i}\right]$ we repeat this process in order to obtain a symmetric curve.

Let us suppose that we have done the same construction for each one of the segments $\left[a_{i}, b_{i}\right]$. Thus we have defined a new sphere $S^{\prime}$, which defines a new norm in $\mathbb{R}^{2}$; we will denote this norm by $\||\cdot|\|_{\left\{\varepsilon,\left\{c_{i}\right\}\right\}}$. Let us note that, in our 


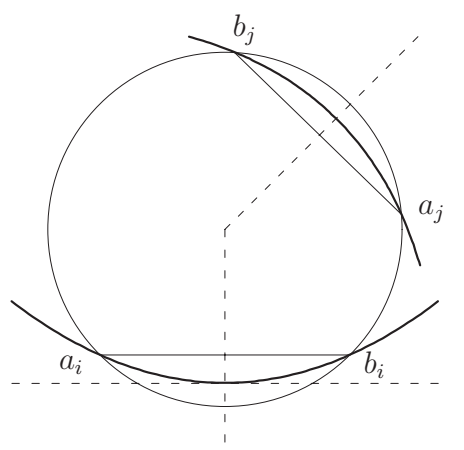

FIGURE 1.

construction, we have chosen parabolic sections instead of arcs from large spheres. It is because the parabolas lend themselves nicely to direct computation.

Remark 2.4. Let us note that, in order to assure that the sphere $S^{\prime}$ defines a new norm, the values $c_{i}$ must satisfy

$$
c_{i} \leq \delta_{E}\left(\varepsilon^{i}\right)=1-\sqrt{1-\frac{\varepsilon^{2 i}}{4}},
$$

where, as before, $\delta_{E}$ is the modulus of convexity of the Euclidean norm. Hence, in particular, for every modulus function $f$ and for every $0<\varepsilon \leq 1 / 2$, we can take $c_{i}=f\left(\varepsilon^{i}\right)$ in the previous construction. In this case, we will denote the resulting norm $\||\cdot|\|_{\{\varepsilon, f\}}$.

The next step is to show the main property of this new norm. In order to do this, we will use the following definition:

Definition 2.5. Given an arbitrary norm $\left\|\cdot|\||\right.$ in $\mathbb{R}^{2}$, we can define the function

$$
\eta(t)=\inf \{\varphi(x, y):\||x|\|=\|\| y\|\|=1,\|x-y\| \geq t\},
$$

where $\varphi(x, y)=\left\|\frac{x+y}{2}\right\|\left(\frac{1}{\prod \frac{x+y}{2} \|}-1\right)$.

Lemma 2.6. Let $\eta$ be as in Definition 2.5. Then $\eta \sim \delta_{\left(\mathbb{R}^{2},|| \cdot|| \mid\right)}$.

Proof. Trivial.

Proposition 2.7. For every $0<\varepsilon \leq 1 / 2$, and for every modulus function $\delta$, there exists a positive constant $L^{\prime}(\varepsilon)$ such that

$$
\delta\left(\varepsilon^{i}\right) \geq \eta\left(\varepsilon^{i}\right) \geq L^{\prime}(\varepsilon) \delta\left(\varepsilon^{i}\right),
$$

for every $i \in \mathbb{N}$, where $\eta$ is defined using the norm $\||\cdot|\|_{\{\varepsilon, \delta\}}$.

Proof. Let us denote just by $|\|\cdot|\||$, the norm $\|| \cdot \mid \|_{\{\varepsilon, \delta\}}$. Let $i \in \mathbb{N}$ be fixed, and let us take two arbitrary points $x$ and $y$ such that $\||x|\|=\|\mid\| y \|=1$ and $\|x-y\|=\varepsilon^{i}$. We have to distinguish two cases:

(1) Let us suppose that $\|\mid x+y\|\|=\| x+y \|$. Obviously $\|x\|,\|y\| \leq 1$. Then, the parallelogram identity assures that

$$
\|x+y\|^{2}=2\|x\|^{2}+2\|y\|^{2}-\|x-y\|^{2} \leq 4-\varepsilon^{2 i},
$$




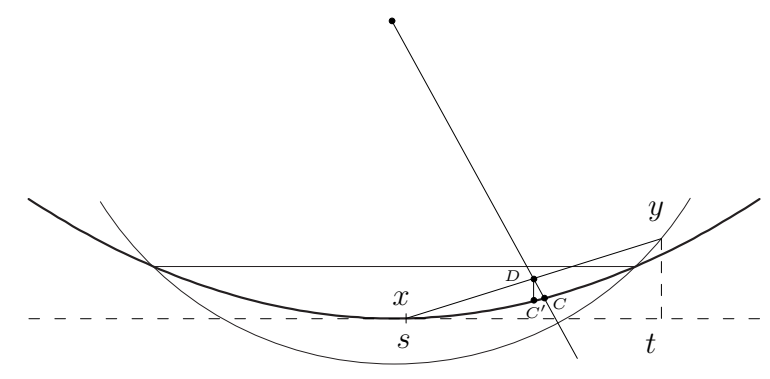

FIGURE 2.

and thus,

$$
\varphi(x, y)=1-\left\|\frac{x+y}{2}\right\| \geq 1-\sqrt{1-\frac{\varepsilon^{2 i}}{4}}=\delta_{E}\left(\varepsilon^{i}\right) \geq \delta\left(\varepsilon^{i}\right) .
$$

(2) Let us suppose that \|\|$x+y\|\| \neq\|x+y\|$. In this case there exists $j \in \mathbb{N}$ such that the point $x+y$ lies inside the cone with the vertex as the origin, and determined by $a_{j}$ and $b_{j}$. If $j>i$, then $\varepsilon^{j}<\varepsilon^{i}$, and then

$$
\begin{aligned}
\varphi(x, y) & \geq \sqrt{1-\frac{\varepsilon^{2 j}}{4}}-\sqrt{1-\frac{\varepsilon^{2 i}}{4}}=\delta_{E}\left(\varepsilon^{i}\right)-\delta_{E}\left(\varepsilon^{j}\right) \\
& \geq \delta_{E}\left(\varepsilon^{i}\right)-\delta_{E}\left(\varepsilon^{i+1}\right) \geq\left(1-\varepsilon^{2}\right) \delta_{E}\left(\varepsilon^{i}\right) \geq\left(1-\varepsilon^{2}\right) \delta\left(\varepsilon^{i}\right) .
\end{aligned}
$$

But if $j \leq i$, we have to be more careful. Let us consider the system of coordinates corresponding to the interval $\left[a_{j}, b_{j}\right]$ that were used to define $\||\cdot|\|$. Let us take the first coordinates, say $t$ and $s$, in this system, of the points $x$ and $y$, respectively. Now, we denote by $D$ the midpoint of the segment $[x, y]$, by $C$ the intersection point of the segment $[0, x+y]$ with the sphere $S^{\prime}$, and by $C^{\prime}$ the orthogonal projection of $D$ on the graph of the function $y_{j}$ (see Figure 2).

Then it holds that $\varphi(x, y)=|D C|$. Clearly,

$$
\left|D C^{\prime}\right| \geq \frac{y_{j}(s)+y_{j}(t)}{2}-y_{j}\left(\frac{s+t}{2}\right)=\frac{\delta\left(\varepsilon^{j}\right)}{\varepsilon^{2 j}}(s-t)^{2},
$$

and we are going to prove that there exists a constant $W(\varepsilon)$ such that $|D C| \geq W(\varepsilon)\left|D C^{\prime}\right|$. In order to show this, we consider the following rightangled triangle: Let us take the straight line passing through $D$, which is perpendicular to the $x$-axis, and the orthogonal line to $D C$ passing through $C$. These two lines intersect in a point that we denote by $G$. Then, we consider the triangle with vertices $D, C$, and $G$. Now, it is clear that $\frac{|D C|}{|D G|}=$ $\cos \alpha$, where $\alpha$ is the angle of our triangle corresponding to the point $D$. But this angle satisfies $|\alpha| \leq \arcsin (\varepsilon / 2)$, and then $\cos \alpha \geq \cos (\arcsin (\varepsilon / 2))$. If we denote by

$$
W(\varepsilon)=\cos \left(\arcsin \frac{\varepsilon}{2}\right),
$$

and note that $|D G| \geq\left|D C^{\prime}\right|$, we finally obtain that $|D C| \geq W(\varepsilon)\left|D C^{\prime}\right|$. 
On the other hand we have that the quotient $|t-s| /\|x-y\|=\cos \beta$, where $\beta$ is the angle determined by the segment $[x, y]$ with respect to the $x$-axis. Since $\varepsilon \leq 1 / 2$ we have that the absolute value $|\beta|$ is always strictly less than $\pi / 2$. Therefore, we can assure that there exists a constant $A(\varepsilon)$ such that $|t-s| \geq A(\varepsilon)\|x-y\|$. Finally, we have that

$$
\begin{aligned}
\varphi(x, y) & =|D C| \geq W(\varepsilon)\left|D C^{\prime}\right| \geq W(\varepsilon) \frac{\delta\left(\varepsilon^{j}\right)}{\varepsilon^{2 j}}(s-t)^{2} \\
& \geq W(\varepsilon) A(\varepsilon)^{2} \frac{\delta\left(\varepsilon^{j}\right)}{\varepsilon^{2 j}}\|x-y\|^{2}=W(\varepsilon) A(\varepsilon)^{2} \delta\left(\varepsilon^{j}\right) \varepsilon^{2(i-j)},
\end{aligned}
$$

and since $\delta$ is a modulus function, we obtain that

$$
\delta\left(\varepsilon^{i}\right)=\delta\left(\varepsilon^{i-j} \varepsilon^{j}\right) \leq L \delta\left(\varepsilon^{j}\right) \varepsilon^{2(i-j)}
$$

for a certain positive constant $L$. Then

$$
\varphi(x, y) \geq \frac{W(\varepsilon) A(\varepsilon)^{2}}{L} \delta\left(\varepsilon^{i}\right)
$$

Now, if we take $L^{\prime}(\varepsilon)=\min \left\{\left(1-\varepsilon^{2}\right), \frac{W(\varepsilon) A(\varepsilon)^{2}}{L}\right\}>0$, we have that

$$
\eta\left(\varepsilon^{i}\right) \geq L^{\prime}(\varepsilon) \delta\left(\varepsilon^{i}\right)
$$

as we want to show.

The first part of the inequality in the theorem is obvious since

$$
\varphi\left(a_{i}, b_{i}\right)=\delta\left(\varepsilon^{i}\right)
$$

2.2. Main Theorem. The main theorem of this work is just a corollary of the previous results. Indeed, we can deduce it from Proposition 2.7.

Theorem 2.8. A function $\delta$ is a modulus function as in Definition 2.2 if and only if it is equivalent, in the sense of Definition 2.1, to the modulus of convexity of a two-dimensional Banach space.

Proof. Let $\delta$ be a modulus function, that is, a non-negative function satisfying the properties in Definition 2.2. If we show that for some $0<\varepsilon<1 / 2$ we have $\eta \sim \delta$, by Lemma 2.6 we have finished the proof. Thus let us fix a certain $0<\varepsilon<1 / 2$. By Proposition 2.7 it holds that

$$
\delta\left(\varepsilon^{i}\right) \geq \eta\left(\varepsilon^{i}\right) \geq L^{\prime} \delta\left(\varepsilon^{i}\right)
$$

for every $i \in \mathbb{N}$.

Let us take an arbitrary $t \in(0, \varepsilon]$ and consider $i$ in such a way that $t \in\left(\varepsilon^{i+1}, \varepsilon^{i}\right]$; then we have

$$
\eta(t) \geq \eta\left(\varepsilon^{i+1}\right) \geq L^{\prime} \delta\left(\varepsilon^{i+1}\right)=L^{\prime} \delta\left(\varepsilon \varepsilon^{i}\right) \geq L^{\prime} \delta(\varepsilon t)
$$

Now, for every fixed $t \in\left(0, \varepsilon^{2}\right]$, there exists $i$ such that $t \in\left(\varepsilon^{i+1}, \varepsilon^{i}\right]$, and finally we have

$$
\eta(t) \leq \eta\left(\varepsilon^{i}\right) \leq \delta\left(\varepsilon^{i}\right)=\delta\left(\varepsilon^{-1} \varepsilon^{i+1}\right) \leq \delta\left(\varepsilon^{-1} t\right) .
$$

Combining (2.1) and (2.2) we conclude that $\eta \sim \delta$. This finishes the proof. 


\section{Applications}

An immediate consequence of our main theorem is that given a uniformly convex Banach space, the set of all moduli of convexity for all equivalent uniformly convex renormings of the space is downward directed. More precisely, we have the following:

Corollary 3.1. Let $(X,\|\cdot\|)$ be a super-reflexive space, let $\delta$ be the modulus of convexity of $\|\cdot\|$, and let $\rho \prec \delta$ be another modulus function. Then there exists an equivalent uniformly convex renorming $\|\cdot|\||$ of $X$ whose modulus of convexity is equivalent to $\rho$.

Proof. Let us consider $Y \subset X$ of codimension 2 and $P: X \rightarrow X$ the projection associated to $Y$ that satisfies $P(X)=Y$. Now, we apply Theorem 2.8 to the 2dimensional subspace $Z=P^{-1}(0)$, obtaining the norm $|\cdot|$ with modulus of convexity equivalent to $\rho$. Hence, the formula

$$
\left\|\left|\left\|x||^{2}=\right\| P x \|^{2}+\right| x-\left.P x\right|^{2}\right.
$$

defines an equivalent norm on $X$ with the desired property.

This observation, together with J. Borwein and J. Vanderwerff's characterization of spaces with a modulus of convexity of power type 2, allows us to answer a question of Godefroy and Zizler described below that arose from writing their book [2, privately communicated to us by Zizler, and it also points out an error in Asplund's work 1 .

Recall an alternative definition of uniform convexity.

Definition 3.2. Let $\|\cdot\|$ be a norm on a Banach space $X$. We say that $\|\cdot\|$ is uniformly convex (UC) if for every pair of sequences $\left\{x_{n}\right\}_{n},\left\{y_{n}\right\}_{n}$ in $B_{X}$ satisfying

$$
\lim _{n \rightarrow \infty} 2\left(\left\|x_{n}\right\|^{2}+\left\|y_{n}\right\|^{2}\right)-\left\|x_{n}+y_{n}\right\|^{2}=0
$$

we have that $\lim _{n}\left\|x_{n}-y_{n}\right\|=0$.

This definition is very useful in renorming theory due to its homogeneity, which greatly simplifies the necessary calculations. However, this definition is not "completely homogeneous" since we assume that $x_{n}, y_{n} \in B_{X}$ (or more generally, we need boundedness). Theorem 3 from [1] claims that the additional assumption $x_{n}$, $y_{n} \in B_{X}$ is redundant. Godefroy and Zizler, in the course of writing their book [2] have come across this problem and, being unable to verify Asplund's argument (which in fact, is sketched in the local setting, when it is true), asked if the redundancy of boundedness of $x_{n}, y_{n}$ is indeed true. We answer this question in the negative.

Indeed, J. Borwein and J. Vanderwerff obtained the next theorem (whose proof is included here with their kind permission).

Definition 3.3. A norm $\|\cdot\|$ on a Banach space $X$ is said to have power type $p$, for $p$ real and positive, if there exists a positive constant $C$ such that $\delta_{(X,\|\cdot\|)}(\varepsilon) \geq C \varepsilon^{p}$.

Theorem 3.4. Let $(X,\|\cdot\|)$ be a Banach space. Then the following conditions are equivalent:

(i) $\left\|x_{n}-y_{n}\right\| \rightarrow 0$ whenever $2\left(\left\|x_{n}\right\|^{2}+\left\|y_{n}\right\|^{2}\right)-\left\|x_{n}+y_{n}\right\|^{2} \rightarrow 0$.

(ii) $\|\cdot\|$ is $U C$ with modulus of convexity of power type 2 . 
Proof. (i) $\Rightarrow$ (ii): Suppose $\|\cdot\|$ does not have modulus of convexity of power type 2 . Then there exist $u_{n}, v_{n} \in S_{X}$ such that $\left\|u_{n}-v_{n}\right\| \geq \frac{1}{n}$ while $\left\|u_{n}+v_{n}\right\| \geq 2-\frac{\varepsilon_{n}}{n^{2}}$ where $\varepsilon_{n} \rightarrow 0^{+}$. Now let $x_{n}=n u_{n}, y_{n}=n v_{n}$; then

$$
\left\|x_{n}-y_{n}\right\| \geq 1 \text { and }\left\|x_{n}+y_{n}\right\| \geq 2 n-\frac{\varepsilon_{n}}{n} .
$$

Consequently,

$$
\begin{aligned}
2\left(\left\|x_{n}\right\|^{2}+\left\|y_{n}\right\|^{2}\right)-\left\|x_{n}+y_{n}\right\|^{2} & \leq 4 n^{2}-\left(2 n-\frac{\varepsilon_{n}}{n}\right)^{2} \\
& =4 \varepsilon_{n}-\frac{\varepsilon_{n}^{2}}{n^{2}},
\end{aligned}
$$

and so $2\left(\left\|x_{n}\right\|^{2}+\left\|y_{n}\right\|^{2}\right)-\left\|x_{n}+y_{n}\right\|^{2} \rightarrow 0$, which means (i) fails.

(ii) $\Rightarrow(\mathrm{i})$ : Suppose $\|\cdot\|$ has modulus of convexity of power type 2 . Now suppose that $\left\|x_{n}-y_{n}\right\| \geq \delta$ but $2\left(\left\|x_{n}\right\|^{2}+\left\|y_{n}\right\|^{2}\right)-\left\|x_{n}+y_{n}\right\|^{2} \rightarrow 0$. Then $\left(\left\|x_{n}\right\|-\left\|y_{n}\right\|\right)^{2} \rightarrow$ 0 . Let $\left\|x_{n}\right\|=\alpha_{n}$. Because $\|\cdot\|$ is uniformly convex, we know $\alpha_{n} \rightarrow \infty$. Thus by replacing $y_{n}$ with $\alpha_{n} \frac{y_{n}}{\left\|y_{n}\right\|}$, we have

$$
\liminf \left\|x_{n}-y_{n}\right\| \geq \delta \text { and }\left\|y_{n}\right\|=\left\|x_{n}\right\|=\alpha_{n} .
$$

Thus we may assume that $\left\|x_{n}-y_{n}\right\|>\eta$ for all $n$ where $\eta=\frac{\delta}{2}$. Now let $u_{n}=\frac{x_{n}}{\alpha_{n}}$ and $v_{n}=\frac{y_{n}}{\alpha_{n}}$. Then $\left\|u_{n}-v_{n}\right\| \geq \frac{\eta}{\alpha_{n}}$, and because the modulus of convexity of $\|\cdot\|$ is of power type 2 , there is a $C>0$ so that $\left\|u_{n}+v_{n}\right\| \leq 2-C\left(\frac{\eta}{\alpha_{n}}\right)^{2}$. Therefore, $\left\|x_{n}+y_{n}\right\| \leq 2 \alpha_{n}-\frac{C \eta^{2}}{\alpha_{n}}$. Consequently, we compute

$$
\begin{aligned}
2\left(\left\|x_{n}\right\|^{2}+\left\|y_{n}\right\|^{2}\right)-\left\|x_{n}+y_{n}\right\|^{2} & \geq 4 \alpha_{n}^{2}-\left(2 \alpha_{n}-\frac{C \eta^{2}}{\alpha_{n}}\right)^{2} \\
& =4 C \eta^{2}-\frac{C^{2} \eta^{4}}{\alpha_{n}^{2}} \nrightarrow 0 .
\end{aligned}
$$

This contradiction completes the proof.

Thus by our main theorem, $l_{2}^{2}$ has an equivalent UC renorming that does not have a modulus of convexity of power type 2 . However, being uniformly convex, it does satisfy the alternative UC definition.

\section{REFERENCES}

1. Edgar Asplund, Averaged norms, Israel J. Math. 5 (1967), 227-233. MR0222610 (36:5660)

2. R. Deville, G. Godefroy, and V. Zizler, Smoothness and renormings in Banach spaces, Pitman Monographs and Surveys in Pure and Applied Mathematics, vol. 64, Longman Scientific \& Technical, Harlow, 1993. MR 1211634 (94d:46012)

3. T. Figiel, On the moduli of convexity and smoothness, Studia Math. 56 (1976), no. 2, 121-155. MR0425581 (54:13535)

4. Göte Nordlander, The modulus of convexity in normed linear spaces, Ark. Mat. 4 (1960), 15-17 (1960). MR0140915 (25:4329)

Departamento de Matemáticas, Universidad de Murcia, 30100 Espinardo (Murcia), SPAIN

E-mail address: ajguirao@um.es

Mathematical Institute, AV ČR, Žitná 25, 11567 Praha 1, Czech Republic

E-mail address: hajek@math.cas.cz 